\title{
The Application of Improved Extended Kalman Filter Algorithm in Satellite Attitude Determination
}

\author{
Dan Jianga ${ }^{\mathrm{a}}$, Xinpu Deng, Jing $\mathrm{Wu}^{\mathrm{b}}$, Shaowen Mo, Yuanyuan Yang \\ School of Electrical Science and Engineering, National University of Defense Technology, \\ Changsha 410073, China \\ a email: danjiang01@126.com, bemail: jingwu@nudt.edu.cn
}

\begin{abstract}
Keywords: Attitude Determination; GEKF Algorithm; Error Consistency; Gyro Drift
\end{abstract}
\begin{abstract}
The classical MEKF algorithm represent the estimation error vector in the estimated attitude coordinates, without considering the deviation between the estimated attitude coordinate and the true attitude coordinate. GEKF algorithm used geometric transform introduced error consistent representation method, which solve the error vector representation inconsistency problem caused by the deviation. But the GEKF algorithm only consider the gyro's constant drift, without considering the time related drift of gyro. To solve this problem, this paper proposes an IGEKF algorithm, the algorithm extended state variable dimensions, using the IGEKF algorithm to estimate the gyro constant drift and time related drift. Simulation results show that the IGEKF algorithm for estimation of gyro drift is more accurate than the GEKF algorithm, the filtering precision has improved more than GEKF algorithm.
\end{abstract}

\section{Introduction}

Using star sensors and gyros in combination has been widely used in satellite attitude system, gyro has higher frequencies which can be used to measure the satellite's three-axis angular velocity. We can get the platform's attitude angle by integrating the angular velocity. But the gyro drift enable measurement error accumulated with time, which will seriously affect measurement accuracy. The measurement accuracy of Star sensor is high, because it has no error accumulation and drift phenomenon. But defect exist in the measurement of low frequency [1]. In order to determine the attitude of the satellite, we can use the combination of star sensor and gyro to overcome their respective shortcomings.

The common attitude representation methods are Euler angles, quaternion, rotation vectors and MRPs $[2,3]$. The quaternion is widely used in satellite attitude determination algorithms because it has small dimension and free of singularities. However, the traditional EKF algorithm does not solve the normalized constraint problem of the quaternion. In MEKF algorithm, attitude quaternion is represented by the product of estimated attitude quaternion and a deviation from that estimate. Using the MEKF algorithm can solve the quaternion normalization constraints problems $[4,5]$. The main idea of the MEKF algorithm is use the EKF algorithm to estimate the vector part of quaternion, then use the corrected quaternion to represents the satellite attitude. The advantage of this algorithm lies in the estimated attitude quaternion satisfy both quaternion normalization constraints and can avoid the singular value to emerge. The MEKF algorithm was first applied to the determination of the satellite attitude in 1969 (SPARS) [6], and it is widely used in the satellite attitude determination system later.

However, in practical application, because we do not know the actual attitude of satellite, so the EKF and MEKF algorithm expressed all vectors in the attitude estimation coordinates. Need to pay attention to that there is a deviation between the true attitude coordinates and estimated attitude coordinates. If vectors and its estimated values are expressed in estimation coordinates, a vector offset will emerge due to the deviation between the two coordinate systems. While the deviation value between two coordinates could not be ignored, the estimation precision for EKF and MEKF algorithms will be seriously affected. To solve this problem, Michael s. Andrle introduced an error consistency represent method in attitude determination problem [7, 8]. This method used a 
geometric transformation to represent vector and its' estimation value in a real coordinate. Based on the method proposed a GEKF algorithm for attitude determination system [7].

Because the literature [7] only give an algorithm that consider the constant drift of the gyro. The estimation accuracy will be affected because the gyro drift is compromised by constant drift and time related drift. In this paper, we first introduce error consistency principle, and applied it to in constant drift and time related drift of gyro model. We derived the IGEKF algorithm in a new gyro measurement model and completed the simulation comparison between IGEKF、GEKF and MEKF algorithm.

\section{Mathematical model of attitude determination}

In star sensor and gyro integrated attitude determination algorithm, the gyroscope measurement is used filter the star sensor measurement noisy, star sensor measurement is used to eliminate the gyro drift measurement, so as to achieve high precision attitude data. Attitude kinematics equations are used to describe the relationship between the platform attitude and angular velocity.

\section{Gyro measurement model}

Gyro is usually used to measure the angular velocity of the platform. Ideally, the measured value of the gyro is proportional to the rotational speed of the satellite body with respect to the rotational speed of the inertial system. But in practice, the gyro is affected by its own design, working mechanism, movement environment and so on, it inevitable consists drift and measurement noise.

The gyro measurement model is[9]:

$$
\boldsymbol{\omega}_{g}=\boldsymbol{\omega}+\mathbf{d}+\mathbf{b}+\boldsymbol{\eta}_{v}
$$

where $\boldsymbol{\omega}$ is the true angular velocity, $\mathbf{d}$ is time related drift for gyro, $\mathbf{b}$ is the gyro constant drift, $\boldsymbol{\eta}_{v}$ is the measurement noise, which modeled as zero mean Gauss white noise. d and bsatisfy the following equation

$$
\boldsymbol{k}=-D_{\tau} \mathbf{d}+\boldsymbol{\eta}_{d}, \boldsymbol{k}=\boldsymbol{\eta}_{b}
$$

where $\boldsymbol{\eta}_{d} 、 \boldsymbol{\eta}_{b}$ is zero mean Gauss white noise, is diagonal matrix consisting of correlation time constant.

The noise is written as the following vector

$$
\mathbf{w}=\left[\begin{array}{l}
\boldsymbol{\eta}_{v} \\
\boldsymbol{\eta}_{d} \\
\boldsymbol{\eta}_{b}
\end{array}\right]
$$

Its' covariance is given by $E\left\{\mathbf{w}(t) \mathbf{w}^{T}(\tau)\right\}=\mathbf{Q}(t) \delta(t-\tau)$,

where $\quad \mathbf{Q}(t)=\left[\begin{array}{ccc}\boldsymbol{\sigma}_{\mathbf{v}}^{2} & \mathbf{0}_{3 \times 3} & \mathbf{0}_{3 \times 3} \\ \mathbf{0}_{3 \times 3} & \boldsymbol{\sigma}_{d}^{2} & \mathbf{0}_{3 \times 3} \\ \mathbf{0}_{3 \times 3} & \mathbf{0}_{3 \times 3} & \boldsymbol{\sigma}_{b}^{2}\end{array}\right]$.

\section{Star sensor measurement model}

Star sensor use the star as the measuring datum to get the star sensor's three axis attitude measurement, and the attitude of the satellite body coordinate system is obtained by a transformation. However, in engineering applications, due to the influence of installation errors, the error of star sensor measurement is inevitable, the actual measurement model of star sensor $[10,11]$ is as follows:

$$
\mathbf{b}=\mathbf{A r}+\mathbf{v}
$$

where $\mathbf{b}$ is a vector in the satellite body coordinate system, $\mathbf{r}$ is a vector in reference coordinate system, $\mathbf{A}$ is an attitude rotation matrix from the reference coordinate system to the satellite body coordinate system, $\mathbf{v}$ is the measure error, modeled as zero mean Gauss white noise.

\section{Attitude kinematics equation}

Attitude quaternion can represent the platform's attitude, and it does not appear singularity 
problem in the attitude calculation, so it is widely used in the attitude determination algorithm.

The attitude quaternion is defined as [12]

$$
\mathbf{q}=\left[\begin{array}{ll}
\boldsymbol{\rho}^{\mathbf{T}} & q_{4}
\end{array}\right]^{\mathbf{T}}
$$

where $\boldsymbol{\rho}$ is the vector part for the quaternion, $q_{4}$ is the scalar part for quaternion.

The attitude matrix is represented by quaternion as

$$
\mathbf{A}(\mathbf{q})=\left(q_{4}^{2}-\|\boldsymbol{\rho}\|^{2}\right) \mathbf{I}_{3 \times 3}+2 \boldsymbol{\rho} \boldsymbol{\rho}^{\mathrm{T}}-2 q_{4}[\boldsymbol{\rho} \times]
$$

Using the platform's attitude quaternion $\mathbf{q}$ and the attitude angular velocity $\omega$ to described the attitude kinematics equations [13]

$$
\dot{\mathbf{q}}=\frac{1}{2} \Omega(\boldsymbol{\omega}) \mathbf{q}=\frac{1}{2} \Xi(\mathbf{q}) \boldsymbol{\omega}
$$

Where,

$$
\Xi(\mathbf{q})=\left[\begin{array}{c}
q_{4} \mathbf{I}_{3 \times 3}+[\boldsymbol{\rho} \times] \\
-\boldsymbol{\rho}^{\mathbf{T}}
\end{array}\right], \Omega(\boldsymbol{\omega})=\left[\begin{array}{cc}
-[\boldsymbol{\omega} \times] & \boldsymbol{\omega} \\
-\boldsymbol{\omega}^{\mathbf{T}} & 0
\end{array}\right]
$$

\section{Error consistency representation and GEKF algorithm}

In order to solve the problem of the deviation between the estimated coordinates and the real coordinates, the geometric transformation is used to solve the Inconsistency of the error vector representation. The corresponding GEKF algorithm is an improvement of MEKF algorithm based on the new form of error vector representation.

Principle of error consistency representation

In EKF algorithm, the state variables are defined as fellow:

$$
\Delta \mathbf{x}=\mathbf{x}-\hat{\mathbf{x}}=\left[\begin{array}{lll}
\Delta \mathbf{q}^{T} & \Delta \mathbf{d}^{\mathbf{T}} & \Delta \mathbf{b}^{\mathbf{T}}
\end{array}\right]^{\mathbf{T}}
$$

Where,

$$
\left\{\begin{array}{l}
\Delta \mathbf{q}=\mathbf{q}-\hat{\mathbf{q}} \\
\Delta \mathbf{d}=\mathbf{d}-\hat{\mathbf{d}} \\
\Delta \mathbf{b}=\mathbf{b}-\hat{\mathbf{b}}
\end{array}\right.
$$

According to the principle of optimal estimation, the optimal estimation $\hat{\mathbf{x}}$ of the state variable $\mathrm{x}$ is satisfied:

$$
\min E\left\{\Delta \mathbf{x}^{\mathbf{T}} \Delta \mathbf{x}\right\}
$$

However, due to the vector representation is not consistent, the error vector $\Delta \mathbf{x}$ defined in this way consists deviation, The vector represent inconsistent problem is illustrated below,together with two vectors $\mathbf{b}$ and $\hat{\mathbf{b}}$ in formula $\Delta \mathbf{b}$.

Notes,

$$
\mathrm{d} \mathbf{q}=\mathbf{q} \otimes \hat{\mathbf{q}}^{-1}
$$

As shown in Figure 1 (a) and 1 (b), the vector $\mathbf{b}$ and $\hat{\mathbf{b}}$ are represent the true gyro drift value and the estimated gyro drift respectively. Expressing $\mathbf{b}$ and $\hat{\mathbf{b}}$ in common coordinates, lead to the bias vector error $\mathrm{db}$.Because in the practical application we do not know real satellite attitude, we can only expressed vector $\hat{\mathbf{b}}$ in the estimation coordinates. But vector $\mathbf{b}$ is defined in the real coordinates, expressing $\mathbf{b}$ in the estimation coordinates, the error vector $\Delta \mathbf{b}$ is correlated with the attitude deviation dq. As show in Figure 1 (c) below, error vector $\Delta \mathbf{b}$ could not represent the actual errordb. 


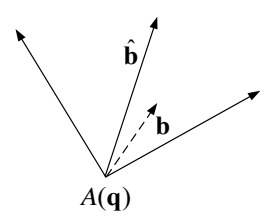

(a) Real

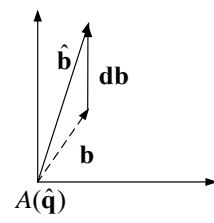

(b) Estimate

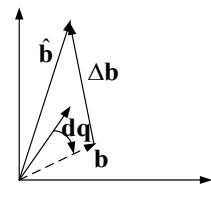

(c) Inconsistency

Fig.1. Inconsistent representation

Denoting two vectors $\mathbf{b}, \mathbf{d}$ and its' error vector consistent representation as db,dd .From Fig. 1(c), using the geometric transformation, the relationship between $\mathrm{db}$ and $\Delta \mathbf{b}$ are defined below:

$$
\mathrm{d} \mathbf{d}=\mathbf{A}(\mathrm{d} \mathbf{q}) \mathbf{d}-\hat{\mathbf{d}}
$$

So as,

$$
\mathrm{d} \mathbf{b}=\mathbf{A}(\mathrm{d} \mathbf{q}) \mathbf{b}-\hat{\mathbf{b}}
$$

Denoted

$$
\mathrm{d} \mathbf{q}=\left[\begin{array}{ll}
\mathrm{d} \mathbf{\rho}^{\mathrm{T}} & \mathrm{d} q_{4}
\end{array}\right]^{\mathbf{T}}
$$

Where, $\mathrm{d} \boldsymbol{\rho}^{\mathrm{T}}$ is the vector part of the error quaternion. When $\mathrm{dq}$ is small, we can get the approximation [14]

$$
\mathrm{d} \boldsymbol{\alpha} \approx 2 \mathrm{~d} \rho
$$

$\mathrm{d} \boldsymbol{\alpha}$ is an attitude angle error vector, attitude rotation matrix can be derived [15],

Define

$$
\mathbf{A}(\mathrm{d} \mathbf{q}):=\mathbf{A}(\mathrm{d} \boldsymbol{\alpha}) \approx \mathbf{I}_{3 \times 3}-[\mathrm{d} \boldsymbol{\alpha} \times]
$$

$$
\mathrm{d} \mathbf{x}=\left[\begin{array}{lll}
\mathrm{d} \boldsymbol{\alpha}^{\mathrm{T}} & \mathrm{d} \mathbf{d}^{\mathrm{T}} & \mathrm{d} \mathbf{b}^{\mathrm{T}}
\end{array}\right]^{\mathbf{T}}
$$

We can derive the following formula:

$$
\Delta \mathbf{x}=\mathbf{C d} \mathbf{x}
$$

where, $\mathbf{C}=\left[\begin{array}{ccc}\frac{1}{2} \Xi(\hat{\mathbf{q}}) & \mathbf{0}_{4 \times 3} & \mathbf{0}_{4 \times 3} \\ {[\hat{\mathbf{d}} \times]} & \mathbf{I}_{3 \times 3} & \mathbf{0}_{3 \times 3} \\ {[\hat{\mathbf{b}} \times]} & \mathbf{0}_{3 \times 3} & \mathbf{I}_{3 \times 3}\end{array}\right]$ called the geometric transformation matrix.

\section{GEKF Algorithm}

Based on the principle of error consistency representation, literature [7] proposed a GEKF algorithm to solve the error represent inconsistency problem between the coordinates and the real coordinates.

\section{(1) State variable and state equation}

In literature[7], the measurement model of gyroscope is modeled as:

$$
\boldsymbol{\omega}_{g}=\boldsymbol{\omega}+\mathbf{b}+\boldsymbol{\eta}_{v}
$$

So the corresponding state variable is:

$$
\mathbf{x}=\left[\begin{array}{ll}
\mathbf{q}^{\mathbf{T}} & \mathbf{b}^{\mathbf{T}}
\end{array}\right]^{\mathbf{T}}
$$

The kinematics equation is approximate to:

$$
\dot{\mathbf{x}} \approx \mathbf{f}(\hat{\mathbf{x}})+\mathbf{F}_{a} \Delta \mathbf{x}+\mathbf{G}_{a} \mathbf{w}
$$

Where, $\mathbf{F}_{a}=\left[\begin{array}{cc}\frac{1}{2} \Omega(\hat{\boldsymbol{\omega}}) & -\frac{1}{2} \Xi(\hat{\mathbf{q}}) \\ \mathbf{0}_{3 \times 4} & \mathbf{0}_{3 \times 3}\end{array}\right], \quad \mathbf{G}_{a}=\left[\begin{array}{cc}-\frac{1}{2} \Xi(\hat{\mathbf{q}}) & \mathbf{0}_{4 \times 3} \\ \mathbf{0}_{3 \times 3} & \mathbf{I}_{3 \times 3}\end{array}\right]$.

In the GEKF algorithm,the error vector is defined by means of the error consistency representation, and the state error vector is defined as : 


$$
\mathrm{d} \mathbf{x}=\left[\begin{array}{ll}
\mathrm{d} \boldsymbol{\alpha}^{T} & \mathrm{~d} \mathbf{b}^{\mathrm{T}}
\end{array}\right]^{\mathbf{T}}
$$

Differential form of the kinematics equation is:

$$
\mathrm{d} \dot{\mathbf{x}}=\mathbf{F}_{g} \mathrm{~d} \mathbf{x}+\mathbf{G}_{g} \mathbf{w}
$$

Where,

$$
\mathbf{F}_{g}=\left[\begin{array}{cc}
-[\tilde{\boldsymbol{\omega}} \times] & -\mathbf{I}_{3 \times 3} \\
{[\hat{\mathbf{b}} \times][\tilde{\boldsymbol{\omega}} \times]} & {[\hat{\mathbf{b}} \times]}
\end{array}\right], \mathbf{G}_{g}=\left[\begin{array}{cc}
-\mathbf{I}_{3 \times 3} & \mathbf{0}_{3 \times 3} \\
{[\hat{\mathbf{b}} \times]} & \mathbf{I}_{3 \times 3}
\end{array}\right]
$$

(2) Measurement variables and measurement equation

Taking the optical axis vector output of two star sensors as the measurement

$$
\mathbf{Z}=\left[\begin{array}{ll}
\mathbf{r}_{1}^{\mathbf{T}} & \mathbf{r}_{2}^{\mathbf{T}}
\end{array}\right]^{\mathbf{T}}
$$

So the measurement equation is as follows:

$$
\mathbf{z}_{k}=h_{k}\left(\mathbf{x}_{k}\right)+\mathbf{v}_{k}
$$

Where, $h_{k}\left(\mathbf{x}_{k}\right)=\mathbf{A}\left(\mathbf{q}_{k}\right) \mathbf{r}_{k}$.

(3) Filtering algorithm

1) Prediction

The state transition matrix for MEKF algorithm $\mathbf{F}_{\text {MEKF }}$ is

$$
\mathbf{F}_{\text {MEKF }}=\left[\begin{array}{cc}
-[(\tilde{\boldsymbol{\omega}}-\hat{\mathbf{b}}) \times] & -\mathbf{I}_{3 \times 3} \\
\mathbf{0}_{3 \times 3} & \mathbf{0}_{3 \times 3}
\end{array}\right]
$$

where oris the output of the gyro's measure velocity.

The differential form of the state transition matrix is obtained by discretization.

$$
\boldsymbol{\Phi}_{M E K F}=\mathbf{I}_{6 \times 6}+\mathbf{F} \Delta t+\mathbf{F}^{2} \Delta t^{2}
$$

where $\Delta t$ Sampling period.

The state transition matrix of the GEKF algorithm is:

$$
\boldsymbol{\Phi}_{k}=\mathbf{T}^{-1} \boldsymbol{\Phi}_{\text {MEKF }} \mathbf{T}
$$

where,

$$
\mathbf{T}=\left[\begin{array}{cc}
\mathbf{I}_{3 \times 3} & \mathbf{0}_{3 \times 3} \\
{[\hat{\mathbf{b}} \times]} & \mathbf{I}_{3 \times 3}
\end{array}\right], \mathbf{T}^{-1}=\left[\begin{array}{cc}
\mathbf{I}_{3 \times 3} & \mathbf{0}_{3 \times 3} \\
{[\hat{\mathbf{b}} \times]^{\mathbf{T}}} & \mathbf{I}_{3 \times 3}
\end{array}\right]
$$

The prediction of the state variable is

$$
\hat{\mathbf{x}}_{k / k-1}=\boldsymbol{\Phi}_{k} \hat{\mathbf{x}}_{k-1}
$$

Prediction error covariance matrix $\mathbf{P}_{k / k-1}$ can use (33) to calculate.

2) Gain

$$
\mathbf{P}_{k / k-1}=\boldsymbol{\Phi}_{k} \mathbf{P}_{k-1} \boldsymbol{\Phi}_{k}^{\mathbf{T}}+\mathbf{T} \mathbf{Q}_{k-1} \mathbf{T}^{\mathbf{T}}
$$

Where,

$$
\mathbf{K}_{k}=\mathbf{P}_{k / k-1} \overline{\mathbf{H}}_{k}^{\mathbf{T}}\left(\overline{\mathbf{H}}_{k} \mathbf{P}_{k / k-1} \overline{\mathbf{H}}_{k}^{\mathbf{T}}+\mathbf{R}_{k}\right)^{-1}
$$

3) Update

$$
\begin{gathered}
\overline{\mathbf{H}}_{k}=\mathbf{H}_{k} \mathbf{C}_{k / k-1}, \\
\mathbf{H}_{k}=\left[\begin{array}{cc}
2\left[\mathbf{A}\left(\hat{\mathbf{q}}_{k / k-1}\right) \mathbf{r}_{k 1} \times\right] \Xi\left(\hat{\mathbf{q}}_{k / k-1}\right) & \mathbf{0}_{3 \times 3} \\
2\left[\mathbf{A}\left(\hat{\mathbf{q}}_{k / k-1}\right) \mathbf{r}_{k 2} \times\right] \Xi\left(\hat{\mathbf{q}}_{k / k-1}\right) & \mathbf{0}_{3 \times 3}
\end{array}\right]
\end{gathered}
$$

State variable update:

$$
\mathrm{d} \hat{\mathbf{x}}_{k}=\mathbf{C}_{k / k-1} \mathbf{K}_{k}\left(\mathbf{z}_{k}-h_{k}\left(\hat{\mathbf{x}}_{k / k-1}\right)\right)
$$




$$
\hat{\mathbf{x}}_{k}=\hat{\mathbf{x}}_{k / k-1}+\mathrm{d} \hat{\mathbf{x}}_{k}
$$

Attitude quaternion normalization:

$$
\hat{\mathbf{q}}_{k}=\frac{\hat{\mathbf{q}}_{k}}{\left\|\hat{\mathbf{q}}_{k}\right\|}
$$

The update value of the error covariance is

$$
\mathbf{P}_{k}=\overline{\mathbf{M}}_{k}\left(\mathbf{I}_{6 \times 6}-\mathbf{K}_{k} \mathbf{H}_{k}\right) \mathbf{P}_{k / k-1} \overline{\mathbf{M}}_{k}^{\mathbf{T}}
$$

where,

$$
\begin{aligned}
& \overline{\mathbf{M}}_{k}=\left(\mathbf{C}_{k}^{\mathrm{T}} \mathbf{C}_{k}\right)^{-1} \mathbf{C}_{k}^{\mathrm{T}} \mathbf{C}_{k / k-1} \\
& =\left[\begin{array}{cc}
\Xi^{\mathrm{T}}\left(\hat{\mathbf{q}}_{k}\right) \Xi\left(\hat{\mathbf{q}}_{k / k-1}\right) & \mathbf{0}_{3 \times 3} \\
{\left[\hat{\mathbf{b}}_{k / k-1} \times\right]-\left[\hat{\mathbf{b}}_{k} \times\right] \Xi^{\mathrm{T}}\left(\hat{\mathbf{q}}_{k}\right) \Xi\left(\hat{\mathbf{q}}_{k / k-1}\right)} & \mathbf{I}_{3 \times 3}
\end{array}\right]
\end{aligned}
$$

\section{Improved GEKF Algorithm}

Due to the drift of gyro in the literature [7], only the constant drift is considered, but gyro also contains the time dependent drift, that is, according to the formula (1) to establish the gyro measurement model. In this paper, the improved GEKF algorithm is based on the new gyro measurement model, and the corresponding formula is derived as follows:

\section{State variable and state equation}

The state variables of the formula (21) are extended, that is

$$
\mathbf{x}=\left[\begin{array}{lll}
\mathbf{q}^{\mathbf{T}} & \mathbf{d}^{\mathbf{T}} & \mathbf{b}^{\mathbf{T}}
\end{array}\right]^{\mathbf{T}}
$$

Kinematic equation is similar to formula (22), where,

$$
\mathbf{F}_{a}=\left[\begin{array}{ccc}
\frac{1}{2} \Omega(\hat{\boldsymbol{\omega}}) & -\frac{1}{2} \Xi(\hat{\mathbf{q}}) & -\frac{1}{2} \Xi(\hat{\mathbf{q}}) \\
\mathbf{0}_{3 \times 4} & -D_{\tau} & \mathbf{0}_{3 \times 3} \\
\mathbf{0}_{3 \times 4} & \mathbf{0}_{3 \times 3} & \mathbf{0}_{3 \times 3}
\end{array}\right], \mathbf{G}_{a}=\left[\begin{array}{ccc}
-\frac{1}{2} \Xi(\hat{\mathbf{q}}) & \mathbf{0}_{4 \times 3} & \mathbf{0}_{4 \times 3} \\
\mathbf{0}_{3 \times 3} & \mathbf{I}_{3 \times 3} & \mathbf{0}_{3 \times 3} \\
\mathbf{0}_{3 \times 3} & \mathbf{0}_{3 \times 3} & \mathbf{I}_{3 \times 3}
\end{array}\right] .
$$

In the IGEKF algorithm, the error vector is defined by means of the error consistency representation, and the state error vector is defined as the error vector:

$$
\mathrm{d} \mathbf{x}=\left[\begin{array}{lll}
\mathrm{d} \boldsymbol{\alpha}^{\mathrm{T}} & \mathrm{d} \mathbf{d}^{\mathrm{T}} & \mathrm{d} \mathbf{b}^{\mathrm{T}}
\end{array}\right]^{\mathrm{T}}
$$

Differential form of the kinematics equation is:

$$
\mathrm{d} \dot{\mathbf{x}}=\mathbf{F}_{g} \mathrm{~d} \mathbf{x}+\mathbf{G}_{g} \mathbf{w}
$$

where,

$$
\begin{gathered}
\mathbf{F}_{g}=\left[\begin{array}{ccc}
-[\hat{\mathbf{d}} \times]-[\hat{\mathbf{b}} \times]-[\tilde{\boldsymbol{\omega}} \times] & -\mathbf{I}_{3 \times 3} & -\mathbf{I}_{3 \times 3} \\
{[\hat{\mathbf{d}} \times][\hat{\mathbf{d}} \times]+[\hat{\mathbf{d}} \times][\hat{\mathbf{b}} \times]+[\hat{\mathbf{d}} \times][\tilde{\boldsymbol{\omega}} \times]} & {[\hat{\mathbf{d}} \times]-D_{\tau}} & {[\hat{\mathbf{d}} \times]} \\
{[\hat{\mathbf{b}} \times][\hat{\mathbf{d}} \times]+[\hat{\mathbf{b}} \times][\hat{\mathbf{b}} \times]+[\hat{\mathbf{b}} \times][\tilde{\boldsymbol{\omega}} \times]} & {[\hat{\mathbf{b}} \times]} & {[\hat{\mathbf{b}} \times]}
\end{array}\right] \\
\mathbf{G}_{g}=\left[\begin{array}{lll}
-\mathbf{I}_{3 \times 3} & \mathbf{0}_{3 \times 3} & \mathbf{0}_{3 \times 3} \\
{[\hat{\mathbf{d}} \times]} & \mathbf{I}_{3 \times 3} & \mathbf{0}_{3 \times 3} \\
{[\hat{\mathbf{b}} \times]} & \mathbf{0}_{3 \times 3} & \mathbf{I}_{3 \times 3}
\end{array}\right]
\end{gathered}
$$

Measurement variables and measurement equation is like formula (26) and (27). 


\section{Filtering Algorithm}

(1) Prediction

The state transition matrix of MEKF algorithm $\mathbf{F}_{M E K F}$ is

$$
\mathbf{F}_{\text {MEKF }}=\left[\begin{array}{ccc}
-[(\tilde{\boldsymbol{\omega}}-\hat{\mathbf{b}}-\hat{\mathbf{d}}) \times] & -\mathbf{I}_{3 \times 3} & -\mathbf{I}_{3 \times 3} \\
\mathbf{0}_{3 \times 3} & D_{\tau} & \mathbf{0}_{3 \times 3} \\
\mathbf{0}_{3 \times 3} & \mathbf{0}_{3 \times 3} & \mathbf{0}_{3 \times 3}
\end{array}\right]
$$

where \&xis the output of the gyroscope measurement.

The differential form of the state transition matrix is obtained by discretization.

$$
\boldsymbol{\Phi}_{\text {MEKF }}=\mathbf{I}_{9 \times 9}+\mathbf{F} \Delta t+\mathbf{F}^{2} \Delta t^{2}
$$

where $\Delta t$ Sampling period.

The state transition matrix of the improved GEKF algorithm is:

$$
\boldsymbol{\Phi}_{k}=\mathbf{T}^{-1} \boldsymbol{\Phi}_{\text {MEKF }} \mathbf{T}
$$

where,

$$
\begin{aligned}
\mathbf{T} & =\left[\begin{array}{ccc}
\mathbf{I}_{3 \times 3} & \mathbf{0}_{3 \times 3} & \mathbf{0}_{3 \times 3} \\
{[\hat{\mathbf{d}} \times]} & \mathbf{I}_{3 \times 3} & \mathbf{0}_{3 \times 3} \\
{[\hat{\mathbf{b}} \times]} & \mathbf{0}_{3 \times 3} & \mathbf{I}_{3 \times 3}
\end{array}\right] \\
\mathbf{T}^{-1} & =\left[\begin{array}{ccc}
\mathbf{I}_{3 \times 3} & \mathbf{0}_{3 \times 3} & \mathbf{0}_{3 \times 3} \\
{[\hat{\mathbf{d}} \times]^{\mathbf{T}}} & \mathbf{I}_{3 \times 3} & \mathbf{0}_{3 \times 3} \\
{[\hat{\mathbf{b}} \times]^{\mathbf{T}}} & \mathbf{0}_{3 \times 3} & \mathbf{I}_{3 \times 3}
\end{array}\right]
\end{aligned}
$$

Predictive value of state variable can be calculated by formula (32), Prediction error covariance matrix $\mathbf{P}_{k / k-1}$ can be calculated by formula (33), where $\mathbf{T}$ and $\mathbf{T}^{-1}$ can be replaced by formula (49) and (50).

(2) Gain

Kalman gain:

where,

$$
\mathbf{K}_{k}=\mathbf{P}_{k / k-1} \overline{\mathbf{H}}_{k}^{\mathbf{T}}\left(\overline{\mathbf{H}}_{k} \mathbf{P}_{k / k-1} \overline{\mathbf{H}}_{k}^{\mathbf{T}}+\mathbf{R}_{k}\right)^{-1}
$$

$$
\begin{gathered}
\overline{\mathbf{H}}_{k}=\mathbf{H}_{k} \mathbf{C}_{k / k-1}, \\
\mathbf{H}_{k}=\left[\begin{array}{cc}
2\left[\mathbf{A}\left(\hat{\mathbf{q}}_{k / k-1}\right) \mathbf{r}_{k 1} \times\right] \Xi\left(\hat{\mathbf{q}}_{k / k-1}\right) & \mathbf{0}_{3 \times 6} \\
2\left[\mathbf{A}\left(\hat{\mathbf{q}}_{k / k-1}\right) \mathbf{r}_{k 2} \times\right] \Xi\left(\hat{\mathbf{q}}_{k / k-1}\right) & \mathbf{0}_{3 \times 6}
\end{array}\right]
\end{gathered}
$$

(3) Update

The state variables increment and state variables of attitude updating, attitude quaternion normalization can be calculated by formulate (36) (37) (38).

The update of the error covariance is

$$
\mathbf{P}_{k}=\overline{\mathbf{M}}_{k}\left(\mathbf{I}_{9 \times 9}-\mathbf{K}_{k} \mathbf{H}_{k}\right) \mathbf{P}_{k / k-1} \overline{\mathbf{M}}_{k}^{\mathbf{T}}
$$

where, 


$$
\begin{aligned}
& \overline{\mathbf{M}}_{k}=\left(\mathbf{C}_{k}^{\mathbf{T}} \mathbf{C}_{k}\right)^{-1} \mathbf{C}_{k}^{\mathbf{T}} \mathbf{C}_{k / k-1} \\
& =\left[\begin{array}{ccc}
\Xi^{\mathbf{T}}\left(\hat{\mathbf{q}}_{k}\right) \Xi\left(\hat{\mathbf{q}}_{k / k-1}\right) & \mathbf{0}_{3 \times 3} & \mathbf{0}_{3 \times 3} \\
{\left[\hat{\mathbf{d}}_{k / k-1} \times\right]-\left[\hat{\mathbf{d}}_{k} \times\right] \Xi^{\mathbf{T}}\left(\hat{\mathbf{q}}_{k}\right) \Xi\left(\hat{\mathbf{q}}_{k / k-1}\right)} & \mathbf{I}_{3 \times 3} & \mathbf{0}_{3 \times 3} \\
{\left[\hat{\mathbf{b}}_{k / k-1} \times\right]-\left[\hat{\mathbf{b}}_{k} \times\right] \Xi^{\mathbf{T}}\left(\hat{\mathbf{q}}_{k}\right) \Xi\left(\hat{\mathbf{q}}_{k / k-1}\right)} & \mathbf{0}_{3 \times 3} & \mathbf{I}_{3 \times 3}
\end{array}\right]
\end{aligned}
$$

\section{Simulation analysis}

For this simulation is based on IGEKF algorithm, the satellite platform's attitude is obtained by using the IGEKF algorithm to processed the star sensor and gyros' measurement. And the simulation results were compared with MEKF and GEKF algorithm.

\section{Parameter setting}

Sensor measurement noise and initial value of the filter as shown in table 1:

Table 1 algorithm parameter settings

\begin{tabular}{|c|c|}
\hline Star Sensor & Measure noise $\sigma_{\mathrm{v}}=1^{\prime \prime}$, Data update frequency $4 \mathrm{HZ}$ \\
\hline Gyro & $\begin{array}{c}\text { Measure noise } \sigma_{b}=0.1^{\prime \prime} / \mathrm{s}, \sigma_{d}=0.01^{\prime \prime} / \mathrm{s}^{2}, D_{\tau}=1 / 3600 \mathbf{I}_{3 \times 3} / \mathrm{s} \\
\text { Data update frequency } 8 \mathrm{~Hz}\end{array}$ \\
\hline Initialization & $\mathbf{Q}=\operatorname{diag}\left(\sigma_{v}^{2} \mathbf{I}_{3 \times 3}, \sigma_{d}^{2} \mathbf{I}_{3 \times 3}, \sigma_{b}^{2} \mathbf{I}_{3 \times 3}\right)$ \\
& $\mathbf{R}=2.5 \times 10^{-12} \mathbf{I}_{6 \times 6}, \mathbf{P}_{0}=2.35 \times 10^{-11} \mathbf{I}_{9 \times 9}$ \\
\hline
\end{tabular}

\section{Simulation analysis}

This simulations are performed using MEKF 、 GEKF and IGEKF algorithm respectively, take 1000 Monte-Carlo simulation results for statistics, the root mean square error of the different algorithms, the corresponding attitude angle estimation and gyro drift estimation.

Table 2 shows the root mean square error of the attitude angle of the different algorithms.

Table 2 different algorithms of RMSE

\begin{tabular}{|c|l|c|c|}
\hline & \multicolumn{3}{|l|}{ Attitude Angle RMSE (") } \\
\hline Algorithm & Roll & Pitch & Yaw \\
\hline Measure & 2.2407 & 1.0964 & 1.4689 \\
\hline MEKF & 0.3601 & 0.3390 & 0.3631 \\
\hline GEKF & 0.2678 & 0.2142 & 0.2108 \\
\hline IGEKF & 0.2380 & 0.1771 & 0.2040 \\
\hline
\end{tabular}

As can be seen from table 2, under the same simulation conditions, compared with the MEKF algorithm ,the RMES for the GEKF algorithm is significantly lower than the GEKF. Compared with GEKF algorithm, the attitude angle estimation accuracy of IGEKF algorithm is improved.

Figure 2 gives the attitude angle estimation for different algorithms.

Figure 3 gives the attitude angle estimation error for different algorithms.

From Figure 2 we can see all the three algorithms can estimate the platform attitude angle, and figure 3 shows the three algorithms of attitude angle estimation error, as can be seen, the GEKF algorithm and IGEKF algorithm can make the attitude angle estimation error within $\pm 0.6^{\prime \prime}$, and IGEKF algorithm can reduce the GEKF algorithm's estimation error in some extent, and use MEKF algorithm of the estimation error is fluctuated within $\pm 1.2^{\prime \prime}$, filtering effect is a bit poor.

The gyro drift estimation of different algorithms is given in Figure 4.

As can be seen from Figure 4, the GEKF algorithm has a great improvement on the gyro drift estimation compared to the MEKF, the IGEKF algorithm is more accurate than the GEKF algorithm for the estimation of the drift. The MEKF algorithm has some deviations in the gyro's drift estimation, especially the drift rate is very small, and the estimated performance will be severely degraded. 

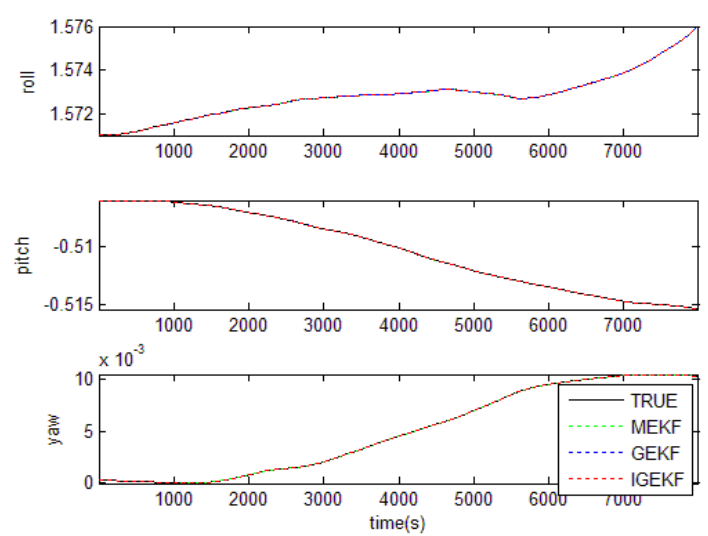

Fig.2. Attitude Angle Estimation
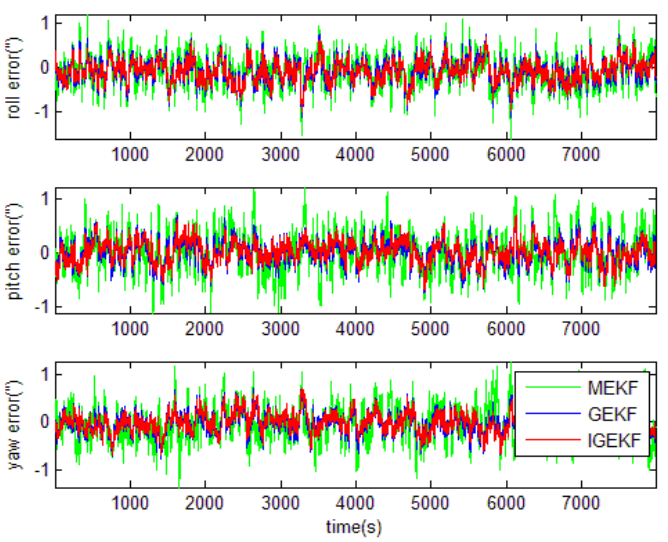

Fig.3. Attitude Angle Estimation Error
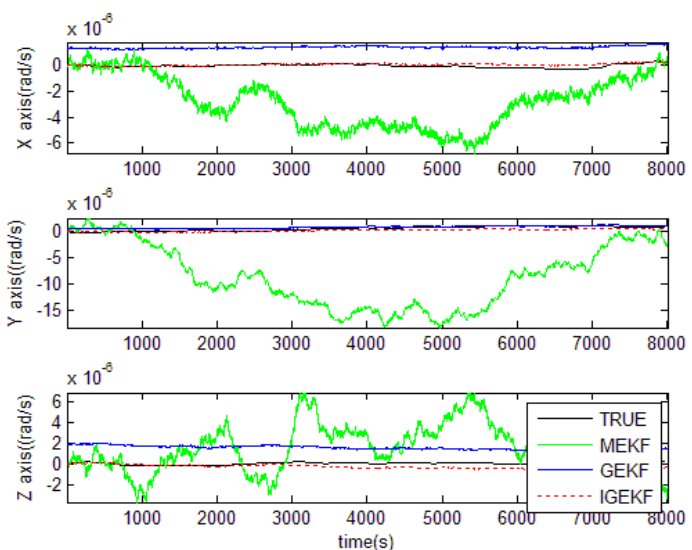

Fig.4. Gyro Drift Estimation

\section{Conclusion}

In this paper, to deal with the problem that error vector representation is not consistent in traditional MEKF algorithm. We applied the GEKF algorithm to the satellite attitude determination system and make some improvements in order to estimate the gyro's time related drift. First, extending the state variables of the GEKF algorithm. Next, using a geometric transform to solve the vector error inconsistency representation. Then take the MEKF to estimate the attitude quaternion increments、gyro constant drift increments and time related drift increments. Finally, using attitude correction to achieve the satellite attitude. Simulation results show that the IGEKF algorithm proposed in this paper can estimate the gyro's constant drift and time related drift accurately, and the filter accuracy has improved significantly compared with the GEKF algorithm.

\section{Acknowledgement}

In this paper, the research was sponsored by the Nature Science Foundation of National (Project No. 61401474).

\section{References}

[1] Lv Zhen-duo, Lei Yong-jun. Satellite Attitude Measurement and Determination [M]. National Defense Industry Press.2013.

[2] Crassidis,J.L, Markley, F.L., and Cheng, Y.A Survey of Attitude Estimation Methods[J].Journal of Guidance, Control, and Dynamics, Vol. 30, No. 1, Jan.-Feb. 2007.

[3] Karlgaard, C.D. and Schaub, H. Nonsingular Attitude Filtering Using Modified Rodrigues 
Parameters [J] .The Journal of the Astronautically Sciences, Vol. 57,No. 4, 2009.

[4] Markley, FL and Crassidis, JL. Fundamentals of Spacecraft Attitude Determination and Control [M] Springer. New York, NY, 2014.

[5] Farrenkopf, R.L. Analytic steady-state accuracy solutions for two common spacecraft attitude estimators [J] .J. Guid. Contr. 1(4), 282-284 (1978) .

[6] Zhou Hai-yin, Wang Jiong-qi, Pan Xiao-gang, Jiao Yuan-yuan. Fusion Theory and Methods for Satellite State Estimation [M] .Science Press.2013.

[7] Michael S,Andrle, John L.Crassidis. Attitude Estimation Employing Common Frame Error Representations [J]. Journal of Guidance Control and Dynamics, August 2015.

[8] Michael S.Andrle, John L.Crassidis. Geometric Integration of Quaternions [J]. Journal of Guidance Control and Dynamics, November 2013.

[9] Markley, F.L. Attitude Error Representations for Kalman Filtering [J], Journal of Guidance, Control and Dynamics, Vol. 26,No. 2, 2003.

[10] Shuster, M. D. Kalman Filtering of Spacecraft Attitude and the QUEST Model [J]. Journal of the Astronautical Sciences, Vol. 38, No. 3.

[11] Shuster,M.D and Oh,S.D, Three-Axis Attitude Determination from Vector Observations[J]. Journal of Guidance and Control, Vol. 4, No. 1,1981,pp. 70-77.

[12] Markley, FL and Crassidis, JL. Fundamentals of Spacecraft Attitude Determination and Control [M] Springer. New York, NY, 2014.

[13] Shuster,M.D, A survey of Attitude Representations [J]. Journal of the Astronautical Science, Vol. 41. N0. 4, 1993, 439-517.

[14] Crassidis,J.L and Junkins,J.L, Optimal Estimation of Dynamic Systems[M].CRC Press ,Boca Raton,FL2nd ed,2012.

[15] Bian Zhi-qiang, Cheng Wei-qiang, Xue Xiao-bu, Yu Yong-jiang. Satellite Attitude Determination Algorithm Based on Gyro and Star-sensor [J]. Spacecraft Engineering, 20(2), 2011.

[16] Hughes, P.C, Spacecraft Attitude Dynamics, Dover Publ. 